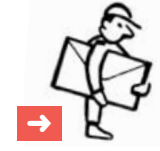

\section{Limitationen unter TARMED}

«Bis dahin kann ich Ihnen nur raten, die Rechnungen nach Ihrer Arbeit mit Limitationen zu stellen.» Das tönt nun (der Unterton wirkt schon fast etwas drohend) schon viel deutlicher als die doch eher schönfärberische Feststellung, fünf Jahre nach Einführung des TARMED seien «Limitationen kein Thema mehr». Es wirkt nicht sehr motivierend, wenn sogar meine Standesvertretung von mir erwartet, klaglos meinen Notfalldienst zu absolvieren und nur für einen Bruchteil der im Einsatz geleisteten Arbeit Rechnung stellen zu dürfen. (In der Randregion eines Kantons praktizierend, der für Eintreffzeiten die Regel «in 30 Minuten 80\% der Bevölkerung erreicht» bereits als Errungenschaft seines Rettungsgesetzes feiert, habe ich ziemlich Erfahrung damit, wie wir Dienstärzte bis zur Ankunft der Rettungsdienste unbezahlt Zeit verrinnen lassen dürfen.)

Davon einmal ganz abgesehen, scheint es mir symptomatisch, wenn fünf Jahre nach Einführung des TARMED «als Nebenschauplatz» «Gespräche» zur Regelung des elektronischen Datenaustausches «anstehen» und man «hofft», «da rasch ans Ziel zu kommen».

Auf allen Ebenen, und notabene auch auf derjenigen der Standesorganisation wird immer wieder die Bedeutung der hausärztlichen Versorgung und speziell des ärztlichen Notfalldienstes betont. Wenn es dann allerdings praktisch wird, «können wir sie leider nicht von den Limitationen entbinden», «kann ich Ihnen nur raten, die Rechnungen [...] mit Limitationen zu stellen», und «muss sich der betroffene Arzt leider selber zur Wehr setzen».

Letzteres habe ich, in der Meinung, die Anwendung von Limitationen sei mindestens für die Tätigkeit im ärztlichen Notfalldienst unzulässig da wir in der Funktion als Dienstarzt nicht die Wahl haben die entsprechenden Leistungen zu erbringen oder nicht-, getan.

Immerhin hat sich die Suva nach Darlegung meiner Sicht, dass es eine Nötigung darstellt, in diesen Fällen Limitationen anzuwenden (gar noch verbunden mit der Aufforderung, für eine direkte elektronische Einlieferung über einen bestimmten Intermediär besorgt zu sein), bequemt, meine Forderung ungekürzt zu überweisen. Die Abteilung für Medizinaltarife in Luzern macht mich darauf aufmerksam, dass sie mich «weiterhin bei künftigen Rechnungen, welche nicht in elektronischer Form bei der Suva eintreffen, nicht von der Limitierung für nichtelektronisch abrechnende Fachärzte entbinden können», was bedeute, dass bei Überschreitung der Limite eine schriftliche Begründung mitgeschickt werden müsse, «um die Mehrverrechnung gemäss GI-1Grundsatz (alle erbrachten Leistungen müssen wirksam, zweckmässig und wirtschaftlich sein) zu rechtfertigen.»

Immerhin wird so ein gewisses Einlenken und eine kurzfristig anwendbare Lösung signalisiert. Allerdings ändert das in meinen Augen nichts daran, dass Limitationen ein Problem darstellen können und umgehend mindestens für die Entschädigung im Notfalldienst langfristige Lösungen ohne unangemessenen bürokratischen Aufwand gefunden werden müssen.

Dr. med. Kaspar Lüthi, Brienz

\section{Replik}

Sehr geehrter Herr Kollege Lüthi

Gespräche und Verhandlungen zum elektronischen Datenaustausch (eDA) haben schon vor der Einführung TARMED stattgefunden. Die Delegationen von santésuisse und KKA haben Verhandlungen zur Umsetzung des elektronischen Datenaustausches geführt und sich für eine datenschutzkonforme Lösung entschieden. Nur hat dann der Verwaltungsrat santésuisse dieser gemeinsamen Vereinbarung der Verhandlungsdelegationen nicht zugestimmt, und damit ist eine Verbandslösung nicht zustande gekommen. Erneute Gespräche auf Verbandsebene sind bis heute immer wieder gescheitert und wurden von den Versicherern immer wieder abgelehnt.

Aus diesem Grund konnten die Krankenkassen auch den im TARMED-Vertrag vorgesehenen obligatorischen eDA zwei Jahre nach Einführung von TARMED nicht durchsetzen.

Lösungen werden aktuell auf Ebene einzelner Versicherer und der Trustcenter gesucht und teilweise auch gefunden. Der eDA im KVG-Bereich 
ist zwischen der Ärzteschaft und einzelnen Versicherern wie VISANA, Groupe mutuel und KPT inzwischen über die Trustcenter als «Intermediär» vertraglich möglich geworden. Leider aber noch nicht mit allen Versicherern. Für die so abrechnenden Ärzte entfallen damit die Limitationen. Im Notfalldienst gelten aber die gleichen Regeln wie im regulären Sprechstundenbetrieb.

Wir können gut verstehen, dass dieser schleppende Verlauf der Verhandlungen mit jedem einzelnen Versicherer über den eDA für die Ärzteschaft unbefriedigend und auf Dauer nicht akzeptabel ist. Andrerseits können wir aber im TG (tiers garant) nur Hand bieten für eine datenschutzkonforme Abwicklung des eDA über einen Intermediär (Trustcenter). Dies wird leider noch nicht von allen Versicherern akzeptiert.

In der 2001 von FMH und Suva (MTK) unterschriebenen Vereinbarung werden die Grundsätze für den eDA geregelt mit dem Ziel, den elektronischen Datenverkehr zu fördern. Begründungen für die Anwendung von Limitationen werden durch die Suva zugelassen. Es gibt nirgends eine
Pflicht zur elektronischen Datenübermittlung. Im Projekt TARMED Suisse 2010, das eine Teilrevision der TARMED-Tarifstruktur zum Ziel hat, ist vorgesehen, in den Kapiteln 00 und 01 unter anderem einen Zeittarif zu verwenden, der keine Limitationen mehr zum Inhalt hat.

Für den Notfalldienst konnten die Notfallinkonvenienzpauschalen in den Verhandlungen mit den Kostenträgern per 1. März 2009 praktisch wieder auf die «alte Abgeltung» zurückgeführt werden.

Ich bin mir bewusst, dass das Problem eDA und Limitationen trotzdem sowohl auf Ebene santésuisse wie auch im UV/MV/IV-(MTK-)Bereich immer noch nicht zufriedenstellend gelöst ist. Ich versichere Ihnen aber, dass wir am Ball bleiben und auf verschiedenen Ebenen langfristige und für beide Seiten befriedigende Lösungen für den eDA und die unseligen Limitationen suchen.

Dr. med. Ernst Gähler, Mitglied des Zentralvorstandes der FMH, Vizepräsident, Leiter Ressort Tarife und Verträge

\section{Briefe an die SÄZ}

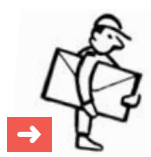

Road-Map für die elektronische Krankengeschichte für die Hausarztpraxis [1]

Ich verstehe die Befürchtungen des Kollegen Reinhardt und bedaure, dass er sich aufgrund eines Missverständnisses derart ärgern musste.

Die Road-Map ist definitiv nicht als Vorschrift für die Ärzteschaft gedacht, sondern als Richtlinie und Gütesiegel für die Software-Hersteller. Das Codiersystem ICPC-2 sollte als Option vorhanden sein, um freiwilligen Teilnehmern Forschung zu ermöglichen.

Warum allerdings die SÄZ den Autoren nicht Gelegenheit zu einer Replik zur Aufklärung des Irrtums in der gleichen Nummer gegeben hat, ist mir unverständlich.

Dr. U. Dürrenmatt, Thun, Arbeitsgruppe SGAM.Informatics

1 Reinhardt U. Road-Map für die elektronische Krankengeschichte für die Hausarztpraxis. Schweiz Ärztezeitung. 2008;89(37):1575-6.
Road-Map für die elektronische Krankengeschichte für die Hausarztpraxis

Sehr geehrter Herr Dr. Reinhardt

Ich habe den Artikel [1] eigentlich nicht so verstanden, dass irgendwer zu einer elektronischen KG gezwungen werden soll. Vielmehr sollen diejenigen, die eine elektronische KG wollen, eine grössere Investitionssicherheit erhalten, indem versucht wird, die Daten transportabel zu machen. Heute ist die Situation nämlich so, dass man mit jedem elektronischen KG-System gewissermassen auf einer Insel sitzt, weil man die darin befindlichen KG-Daten nicht in ein anderes System transferieren kann. Die von Ihnen angesprochene Lösung, dass Arzt A einen Brief ausdruckt, per Post verschickt und Arzt B töggelt oder scannt die darin befindlichen Angaben dann wieder in sein KG-System ein, finde ich eigentlich unnötig kompliziert (wobei ich Ihre Ansicht teile, dass das unkritische Drücken des «Print»Buttons zu unsinnigen Papierfluten führt und der manuellen Zusammenstellung des Relevanten unterlegen ist - diese manuelle Auswahl kann aber auch bei einem elektronischen Transfer gemacht werden). Noch schlimmer ist die Situation, wenn jemand eine Praxis mit elektronischer 
KG übernimmt: Es ist heute praktisch aussichtslos, KG-Daten von einem Programm in ein anderes transferieren zu wollen. Man muss also wohl oder übel das Programm des Vorgängers übernehmen, wenn man nicht einige tausend KGs abtippen (lassen) möchte ... Angesichts des Fortschritts und der unterschiedlichen Entwicklung verschiedener Programme ist das eine unbefriedigende Herstellerbindung.

Ich finde es legitim, hier Standards setzen zu wollen.

Gerry Weirich,

Facharzt für Innere Medizin FMH, Schaffhausen

1 Reinhardt U. Road-Map für die elektronische

Krankengeschichte für die Hausarztpraxis. Schweiz Ärztezeitung. 2008;89(37):1575-6.

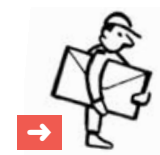

\section{Publicité}

Je sais que les temps sont durs et qu'il faut se lever tôt pour décrocher une pleine page de publicité dans une revue ou un magazine. Mais est-ce une excuse pour tout accepter? Dans le No $35 \mathrm{du}$ BMS du 27 août 2008, en regard de la page 1484 l'excès s'étale avec morgue et impudence: La Cayenne $\mathrm{S}$ Turbo. Combien de médecins suisses vont-ils s'équiper de ce monstre au muffle arrogant (550 chevaux pour faire sa visite hebdomadaire au home ...), polluant de manière indécente (358 g/km de $\left.\mathrm{CO}_{2}\right)$ ? Il ne s'agit en rien de verser dans un militantisme vert angélique mais tout de même, trop c'est trop. C'est un peu comme placarder une affiche vantant un cabaret à strip-tease dans un monastère ...

Prof. A. de Torrenté, La Chaux-de-Fonds

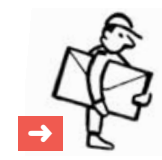

\section{Gedanken zur anstehenden Reduktion der Labortarife und der Vertriebsmarge} Sehr geehrte Bundesräte

Sehr geehrte Politiker

Das Wort «Kostenexplosion» im Gesundheitswesen ist in aller Leute Mund. Hiermit unterbreite ich Ihnen folgende fünf Massnahmen zur nachhaltigen Kosteneindämmung:
1. Der Labortaxpunktwert sollte weiter stufenweise bis auf etwa $40 \%$ des aktuellen Wertes gesenkt werden.

2. Der Röntgentarif sollte weiter gesenkt werden.

3. Die Vertriebsmarge von Medikamenten und Impfungen muss auf 0\% gesenkt werden.

4. Der Taxpunktwert für ärztliche Leistungen sollte ebenfalls sukzessive auf ca. Fr. 0.50 gesenkt werden.

5. Alle Praxen müssen endlich ISO-zertifiziert werden. Ein ausgedehntes Qualitätsmanagement ist schweizweit einzuführen und durch das BAG regelmässig zu Lasten des Praxisinhabers zu überwachen.

Diskussion und Begründung dieser Massnahmen Ad 1: Zum aktuell sehr lukrativen Labortaxpunktwert werden zu viele unnötige Blutuntersuchungen gemacht. Die Praxislabors werfen zu Lasten der Versicherungen einen hohen Gewinn ab.

Die häufigen Quick und Zuckermessungen können nötigenfalls durch den Patienten selbst bestimmt werden. Eine kurze telefonische Besprechung reicht, um ärztliche Instruktionen entgegenzunehmen.

Alle anderen Messungen gehören ins Grosslabor; sei es direkt oder via Spital. Die dort durchgeführten Analysen können zu einem Bruchteil der bisherig vergüteten Kosten durchgeführt werden. Im Zweifelsfall kann der Patient ja auch hospitalisiert werden.

Ad 2: In der Schweiz stehen zu viele Röntgengeräte herum. Wir haben weltweit die höchste Dichte dieser Apparate, die lediglich der Amortisation harren.

Ist ein Röntgenbild zwingend nötig, dann ist der Patient in das nächstgelegene Spital zu schicken. Dadurch kann die dortige Anlage mitsamt dem Personal besser ausgelastet werden. Durch diese Effizienzsteigerung kann bei gleichbleibendem Gewinn der Taxpunktwert etwa halbiert werden. Ad 3: Es ist unethisch, wenn ein Arzt am Vertrieb eines Medikamentes etwas verdient. Mit dem «Kranksein» eines Menschen wird ja auch sonst noch genug Geld gemacht.

Ad 4: Ein Blick in die Einkommensstatistik der Ärzte genügt, um zu sehen, dass eine Taxpunktwertabsenkung noch längst verkraftet werden kann. Zudem würden die Praxen endlich zu einer Effizienzsteigerung gezwungen werden, was eine Taxpunktwertabsenkung zusätzlich rechtfertigen würde.

Ad 5: Bis heute existieren keine verbindlichen Behandlungsstandards nach definierten Qualitätskriterien und Garantieleistungen. Es ist immer noch so, dass jeder Arzt zu Lasten der Krankenversicherungen «wursteln» kann, wie er will. Hier besteht viel Nachholbedarf. 


\section{Zusammenfassung}

Die vorgeschlagenen Sparmassnahmen sollten möglichst zügig umgesetzt werden. Wie die Vergangenheit zeigt, ist von der unter sich zerstrittenen Ärzteschaft kein ernstzunehmender Widerstand zu erwarten.

Durch die Massnahmen würde die Arbeitsmotivation der Ärzteschaft wieder vermehrt weg vom Finanziellen hin zu ideellen Zielen gelenkt.

Gleichzeitig würden auch umweltschützerische Ziele erreicht werden, würde doch der Besitz von Sportwagen, Motorbooten und Zweitwohnungen unter der Ärzteschaft deutlich zurückgehen. Da die Schweiz ja im Vergleich zum Ausland sowieso mit Ärzten überversorgt ist, wäre eine Absenkung der Ärztedichte klar erwünscht, was wiederum mit einer Kosteneindämmung einhergehen würde.

Zwar müssten etliche Patienten deutlich längere Wartezeiten in Kauf nehmen. Wie aber Untersuchungen zeigen, lösen sich viele Probleme in der Medizin innert einer Woche nach Auftreten der Erstsymptome von selbst. Dadurch könnten automatisch viele teure und unnötige Konsultationen gespart werden.

Dr. med. Samuel Gut, Konolfingen

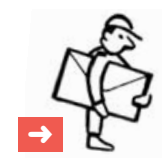

\section{HPV-Impfung? - Voreilig?}

Die kürzlich eingeführte Gratis-HPV-Impfung für junge Mädchen scheint als Hausärzte-Provokations-Impfung in die Schweizer Medizinalgeschichte eingehen zu wollen. Die meisten Grundversorger regen sich mit Recht über die absurde Finanzregelung mit Verlustgarantie auf.
Nicht weniger Grund zum Aufregen gibt mir aber auch die wissenschaftlich unsichere Datenlage bezüglich Wirksamkeit, Wirtschaftlichkeit und Gefahr von langfristig unliebsamen Auswirkungen einer breitgefächerten Impfung. So schreibt eine der renommiertesten Fachzeitschriften, das New England Journal of Medicine, in einem Editorial im August 2008 [1] über die HPV-Impfung: «... How can policymakers make rational choices about the introduction of medical interventions that might do good in the future, but for which evidence is insufficient, especially since we will not know for many years whether the intervention will work or - in the worst case - do harm? ...» Das Editorial schliesst mit den Worten: «... With so many essential questions still unanswered, there is good reason to be cautious about introducing large-scale vaccination programs. Instead, we should concentrate on finding more solid answers through research rather than base consequential and costly decisions on yet unproven assumptions ...»

Voreiliges Handeln ist in der Medizin nicht empfehlenswert. Überlegte Sorgsamkeit ist angebracht!

Dr. Gaston Dunkelmann, Spiez

1 Haug CJ. Human papilloma virus vaccination reasons for caution. $\mathrm{N}$ Engl J Med. 2008;359(8):861-2. 